\title{
Asociación entre los niveles séricos de vitamina D y marcadores inflamatorios en pacientes en hemodiálisis
}

\author{
Hilda M. Villafuerte-Ledesma, * Belén Moragrega, Elena Castillón, Marta Luzón-Alonso y \\ Mercedes García-Mena \\ Hospital San Juan de Dios, Departamento de Nefrología, Zaragoza, España
}

\section{Resumen}

Introducción: La relación entre 25-OH-vitamina $D$ y el sistema inmune en pacientes con enfermedad renal crónica es objeto de atención. Objetivos: Evaluar la prevalencia de la deficiencia de vitamina $D$ en pacientes en hemodiálisis e investigar la asociación entre la vitamina $D$ y proteína C reactiva ultrasensible (PCRus), índice neutrófilo/linfocito (INL) e índice plaqueta/ linfocito (IPL). Método: Estudio transversal de 80 pacientes en hemodiálisis, divididos en dos grupos: un nivel sérico de 25-OH-vitamina $D<20 \mathrm{ng} / \mathrm{mL}$ se consideró como deficiencia de vitamina $D y \geq 20 \mathrm{ng} / \mathrm{mL}$, como normal. Con el análisis de correlación de Spearman se definió la relación entre los parámetros. Resultados: $40 \%$ de los pacientes presentó deficiencia de vitamina $D$. Hubo diferencias significativas entre los grupos en PCRus ( $p=0.047)$, INL $(p=0.039)$, IPL ( $p=0.042)$ y tratamiento con análogos de vitamina $D(p=0.022)$. La vitamina $D$ tuvo una correlación negativa significativa con PCRus $(p=0.026)$, INL $(p=0.013)$ e IPL $(p=0.022)$. Conclusiones: La deficiencia de vitamina $D$ fue de $40 \%$. Los niveles de PCRus, INL e IPL fueron significativamente más altos ante deficiencia de vitamina $D$. Se encontró correlación inversa significativa entre vitamina $D$ y PCRus, INL e IPL.

PALABRAS CLAVE: Deficiencia de vitamina D. Proteína C reactiva ultrasensible. Índice neutrófilo-linfocito. Índice plaqueta-linfocito.

\section{Association between vitamin D serum levels and inflammatory markers in patients on hemodialysis}

\begin{abstract}
Introduction: The relationship between $25-\mathrm{OH}$-vitamin $\mathrm{D}$ and the immune system in patients with chronic kidney disease is a subject of attention. Objectives: To assess the prevalence of vitamin $D$ deficiency in patients on hemodialysis and to investigate the association between vitamin $D$, ultra-sensitive $C$-reactive protein (US-CRP), neutrophil-to-lymphocyte ratio (NLR) and platelet-to-lymphocyte ratio (PLR). Method: Cross-sectional study of 80 patients on hemodialysis, divided into two groups: a serum 25-OH-vitamin D level $<20 \mathrm{ng} / \mathrm{mL}$ was considered to be vitamin $D$ deficiency and a serum level $\geq 20 \mathrm{ng} / \mathrm{mL}$ was regarded as normal. The relationship between the parameters was defined with Spearman's correlation analysis. Results: $40 \%$ of the patients had vitamin $D$ deficiency. There were significant differences between groups in US-CRP $(p=0.047)$, $N L R(p=0.039), P L R(p=0.042)$ and treatment with vitamin $D$ analogues $(p=0.022)$. Vitamin $D$ had a significant negative correlation with US-CRP ( $p=0.026), N L R(p=0.013)$ and PLR $(p=0.022)$. Conclusions: The prevalence of vitamin $D$ deficiency was $40 \%$. The values of US-CRP, NLR and PLR were significantly higher in the presence of vitamin D deficiency. $A$ significant inverse correlation was found between vitamin D levels and US-CRP, NLR and PLR.
\end{abstract}

KEY WORDS: Vitamin D deficiency. Ultra-sensitive C-reactive protein. Neutrophil-to-lymphocyte ratio. Platelet-to-lymphocyte ratio.

Correspondencia:

*Hilda M. Villafuerte-Ledesma*

E-mail: hilda.villafuerte21@gmail.com

0016-3813/๑ 2020 Academia Nacional de Medicina de México, A.C. Publicado por Permanyer. Este es un artículo open access bajo la licencia CC BY-NC-ND (http://creativecommons.org/licenses/by-nc-nd/4.0/).
Fecha de recepción: 02-01-2020

Fecha de aceptación: 23-04-2020

DOI: $10.24875 / G M M .20000004$
Gac Med Mex. 2020;156:519-525

www.gacetamedicademexico.com
Disponible en PubMed 


\section{Introducción}

La deficiencia de vitamina $D$ es altamente prevalente en la población en general; particularmente en la población con enfermedad renal crónica (ERC), la evidencia sugiere que 76 a $92 \%$ la padece. ${ }^{1}$ La deficiencia de vitamina $D$ está asociada con alto recambio óseo, hiperparatiroidismo y baja densidad mineral ósea en pacientes en hemodiálisis $(\mathrm{HD})^{2,3}$ Se ha observado que los niveles de 25-OH-vitamina D (25$\mathrm{OH}-\mathrm{D}) \geq 30 \mathrm{ng} / \mathrm{mL}$ mejoran la fuerza muscular ${ }^{2}$ y los niveles bajos de 25-OH-D se han asociado con disminución de la fuerza y masa muscular, ${ }^{2}$ así como con riesgo de caída. ${ }^{4}$ Por otra parte, los bajos niveles de vitamina $D$ están asociados con síndrome metabólico y obesidad central, ${ }^{5}$ así como con peor función cognitiva en pacientes en HD. ${ }^{6}$

Numerosos estudios publicados en los últimos años han demostrado que existe estrecha asociación entre los niveles reducidos de $25-\mathrm{OH}-\mathrm{D}$ y la mortalidad por todas las causas, así como con la mortalidad cardiovascular en sujetos con ERC, incluidos los pacientes incidentes y prevalentes en HD y diálisis peritoneal, en quienes la inflamación es uno de los principales mecanismos subyacentes a la afección. ${ }^{7-9}$ La inflamación en pacientes en HD puede ser medida por proteína $C$ reactiva (PCR), PCR ultrasensible (PCRus), interleucina-6, factor de necrosis tumoral alfa, procalcitonina, albúmina, ferritina y colesterol. De esos marcadores, la cuantificación de PCRus es el estándar de oro por su bajo costo y disponibilidad. ${ }^{10}$ Recientemente se ha propuesto el índice neutrófilos/ linfocito (INL) y el índice plaqueta/linfocito (IPL) como representación de la inflamación en pacientes con diferentes trastornos, incluidos los que se encuentran en HD. .11,12 $^{2}$

La dirección de la relación entre los niveles de vitamina $D$ y los biomarcadores inflamatorios sigue siendo controvertida. Algunos estudios han afirmado que la inflamación reduce los niveles de vitamina D, mientras que otros indican que el aumento de la vitamina D puede suprimir la inflamación. ${ }^{13,14}$ Característicamente, los pacientes en HD suelen tener niveles bajos de $25-\mathrm{OH}-\mathrm{D}$, sin embargo, pocos estudios han estudiado la relación entre la vitamina D y la inflamación en pacientes en HD. Por lo tanto, los objetivos de este estudio fueron evaluar la prevalencia de deficiencia de vitamina $D$ en pacientes en HD y analizar la asociación entre los niveles de 25$\mathrm{OH}-\mathrm{D}$ y marcadores inflamatorios.

\section{Método}

Estudio transversal realizado en 102 pacientes de la Unidad de Hemodiálisis del Hospital San Juan de Dios de Zaragoza, España, evaluados en agosto de 2019. Los criterios de inclusión fueron edad $\geq 18$ años y en HD crónica por al menos tres meses. Los criterios de exclusión incluyeron ausencia de niveles de vitamina $D$, infección activa, antecedentes de malignidad, enfermedad inflamatoria activa y uso de tratamiento inmunosupresor. En general, cuatro pacientes no tuvieron niveles de 25-OH-D, un paciente fue excluido debido a una infección activa, 13 a malignidad, dos a uso de drogas inmunosupresoras y dos más a enfermedad inflamatoria activa. De los 102 pacientes, 80 cumplieron con los criterios del estudio, el cual fue aprobado por el comité de ética local.

Fueron registradas las características clínicas y demográficas de los pacientes (edad, sexo, tipo de acceso vascular, tiempo en HD, presencia de diabetes mellitus, índice de comorbilidad de Charlson, índice de masa corporal y medicación).

Los datos de laboratorio como 25-OH-D, hemoglobina, leucocitos, albúmina, PCRus, ferritina, PTH, calcio, fósforo, colesterol HDL, colesterol LDL y triglicéridos se obtuvieron de la base de datos de la historia clínica electrónica.

El Kt/v se estimó utilizando la ecuación Daugirdas de segunda generación: ${ }^{15}$

$$
\begin{aligned}
& K t / V=-\operatorname{Ln}[(C 2 / C 1)-(0.008 \times T)]+[4-3.5 \times(C 2 / \\
& \text { C1)] } \times \text { UF/peso }
\end{aligned}
$$

Donde:

C1, C2 = urea inicial y final.

$\mathrm{T}=$ tiempo en horas.

UF = cambio de peso pre-posdiálisis en $\mathrm{kg}$.

Aunque no existe consenso sobre los niveles séricos óptimos de $25-\mathrm{OH}-\mathrm{D}$, la mayoría de los expertos define la deficiencia de vitamina $D$ como un nivel sérico de $25-\mathrm{OH}-\mathrm{D}<20 \mathrm{ng} / \mathrm{mL} .{ }^{16}$ Por lo tanto, en nuestro estudio, un nivel sérico de $25-\mathrm{OH}-\mathrm{D}<20 \mathrm{ng} / \mathrm{mL}$ se identificó como deficiencia de vitamina $D$ y $\geq 20 \mathrm{ng} / \mathrm{mL}$, como normal; sin embargo, se utilizaron las guías Kidney Disease Outcomes and Quality Initiative (KDOQI) para definir el estatus de la vitamina $D$ en los pacientes en HD conforme el nivel sérico de 25-OH-D:

- Deficiencia, < $20 \mathrm{ng} / \mathrm{mL}$.

- Insuficiencia, de 21 a $30 \mathrm{ng} / \mathrm{mL}$.

- Suficiencia, > $30 \mathrm{ng} / \mathrm{mL}$. 
Subcategorizamos a los pacientes con deficiencia severa de vitamina $D$ si el nivel sérico era $<10 \mathrm{ng} / \mathrm{mL}^{17}$

El INL se obtuvo dividiendo el recuento absoluto de neutrófilos entre el recuento absoluto de linfocitos en sangre periférica y el IPL, dividiendo el recuento absoluto de plaquetas entre el recuento absoluto de linfocitos en sangre periférica.

Para la medida de los requerimientos de eritropoyetina se utilizó el índice de resistencia a la eritropoyetina (unidades de eritropoyetina semana/peso en $\mathrm{kg} / \mathrm{hemoglobina} \mathrm{en} \mathrm{g}$ en $100 \mathrm{~mL}$ ).

Para el análisis estadístico se utilizó el programa SPSS en su versión 21.0 para Windows. Se realizó el análisis estadístico descriptivo de cada variable. La mediana y el rango intercuartílico se utilizaron para describir variables cuantitativas con distribución asimétrica. La diferencia estadísticamente significativa entre los grupos se determinó mediante la prueba de $\chi^{2}$ de Pearson (para las variables categóricas) y la prueba $U$ de Mann-Whitney (para las variables continuas sin distribución normal). Las asociaciones entre las variables se exploraron utilizando la rho de Spearman (para datos que no se distribuyen normalmente). Se consideró significativa una $p<0.05$. Se hizo un análisis de subgrupos dividiendo a los pacientes según los niveles de 25-OH-D $(<20 \mathrm{y} \geq 20 \mathrm{ng} / \mathrm{mL}$ ).

\section{Resultados}

Un total de 80 pacientes fueron incluidos en el estudio. La mediana de edad fue de 73.35 años y $62.5 \%$ de los pacientes fue del sexo masculino y la mediana de $25-\mathrm{OH}-\mathrm{D}$ fue de $25.2 \mathrm{ng} / \mathrm{mL}$, como se observa en las características basales de la población de estudio (Tabla 1). Los pacientes fueron divididos en dos grupos: grupo 1 con $25-\mathrm{OH}-\mathrm{D}<20 \mathrm{ng} / \mathrm{mL}$ y grupo 2 con $25-\mathrm{OH}-\mathrm{D} \geq 20 \mathrm{ng} / \mathrm{mL}$. La mediana de 25-OH-D fue de $11.9(9.8-14.5) \mathrm{ng} / \mathrm{mL}$ en el grupo con deficiencia y de $38.6(26.4-56.1) \mathrm{ng} / \mathrm{mL}$ en el grupo con niveles normales. PCRus, INL e IPL presentaron diferencias estadísticamente significativas entre ambos grupos $(p=0.047,0.039$ y 0.042 , respectivamente).

Solo 32 pacientes (40\%) tuvieron nivel suficiente de vitamina D y 16 (20\%), insuficiente. Los 32 pacientes restantes $(40 \%)$ presentaron deficiencia de vitamina D; de ellos, ocho (10\%) tuvieron deficiencia severa $<10 \mathrm{ng} / \mathrm{mL}$ (Tabla 2).

La correlación entre 25-OH-D y otros parámetros fue evaluada mediante análisis de correlación bivariada (Tabla 3). La 25-OH-D presentó una correlación positiva significativa con Kt/v $(r=0.245$, $p=0.034$ ) y una correlación negativa con PCRus, INL e IPL $(r=-0.334, p=0.026 ; r=-0.523, p=0.013 y$ $r=-0.451, p=0.022$, respectivamente). No hubo asociación significativa entre albúmina y niveles de vitamina $D(r=0.003, p=0.98)$. No se halló asociación significativa entre $25-\mathrm{OH}-\mathrm{D}$, calcio, fósforo y hormona paratiroidea $(r=0.197, p=0.08 ; r=-0.063$, $p=0.579$ y $r=-0.093, p=0.411$, respectivamente). Tampoco se encontró asociación significativa entre los requerimientos de eritropoyetina y niveles de vitamina $D(r=-0.045, p=0.694)$.

\section{Discusión}

Los pacientes con ERC en HD tienen niveles séricos elevados de mediadores inflamatorios, particularmente de PCRus, ya que se ha demostrado que desempeñan un papel central en el círculo vicioso de desnutrición, inflamación, aterosclerosis, aumento del riesgo de eventos cardiovasculares y mortalidad general. ${ }^{18}$ En nuestro estudio, en los pacientes también se observó aumento de los marcadores inflamatorios. En los últimos años, INL e IPL se han identificado como marcadores relativamente económicos y universalmente disponibles que pueden usarse para estimar la supervivencia y los resultados adversos en diversas enfermedades, incluida la ERC terminal. ${ }^{13,18}$

La deficiencia de vitamina $D$ (niveles séricos de $25-\mathrm{OH}-\mathrm{D}<20 \mathrm{ng} / \mathrm{mL}$ ) y la insuficiencia (niveles séricos de $25-\mathrm{OH}-\mathrm{D}$ de 21 a $30 \mathrm{ng} / \mathrm{mL}$ ) son comunes en los pacientes con ERC, incluidos los pacientes en HD. En este estudio, la deficiencia e insuficiencia de vitamina $\mathrm{D}$ se presentó en 40 y $20 \%$, respectivamente. La deficiencia severa también fue común: $10 \%$ de los pacientes mostró valores séricos de $25-\mathrm{OH}-\mathrm{D}<$ $10 \mathrm{ng} / \mathrm{mL}$. Nuestros hallazgos son consistentes con datos previos de Marinelli, quien informó $73 \%$ de deficiencia y $23 \%$ de insuficiencia de vitamina $D$ en pacientes en HD. ${ }^{19}$

Se ha descrito una asociación entre la vitamina $D$ y la inflamación, aunque no existe un claro consenso, por ejemplo, Amer et al., en un estudio transversal en 15167 pacientes, observaron una relación inversa estadísticamente significativa entre $25-\mathrm{OH}-\mathrm{D}<21 \mathrm{ng} / \mathrm{mL}$ y PCR. Un estudio retrospectivo en 4120 pacientes reportó una asociación significativa entre INL e IPL con niveles de 25-OH-D. ${ }^{20}$ Por el contrario, en un estudio de pacientes con urticaria crónica no se encontró 
Tabla 1. Características demográficas, clínicas y analíticas de pacientes en hemodiálisis con deficiencia o valores normales de 25-OH-vitamina D

\begin{tabular}{|c|c|c|c|c|}
\hline & Total $(\mathbf{N}=\mathbf{8 0})$ & $\begin{array}{l}\text { Deficiencia de 25-OH-D } \\
\qquad(\mathrm{n}=32)\end{array}$ & $\begin{array}{l}\text { Sin deficiencia de } \\
25-\mathrm{OH}-\mathrm{D}(\mathrm{n}=48)\end{array}$ & $p$ \\
\hline & Mediana (RI) & Mediana (RI) & Mediana (RI) & \\
\hline Edad (años) & 73.35 (57.8-80.1) & 73.5 (56.7-79.9) & $73.2(59-80.4)$ & 0.746 \\
\hline $\begin{array}{l}\text { Sexo } \\
\text { Hombre } \\
\text { Mujer }\end{array}$ & $\begin{array}{l}50(62.5 \%) \\
30(37.5 \%)\end{array}$ & $\begin{array}{l}19(59.4 \%) \\
13(40.6 \%)\end{array}$ & $\begin{array}{l}31(64.6 \%) \\
17(35.4 \%)\end{array}$ & 0.637 \\
\hline $\begin{array}{l}\text { Acceso vascular } \\
\text { Fístula auriculoventricular } \\
\text { Catéter yugular } \\
\text { Catéter femoral }\end{array}$ & $\begin{array}{c}27(33.8 \%) \\
50(62.5 \%) \\
3(3.7 \%)\end{array}$ & $\begin{array}{c}12(37.5 \%) \\
18(56.3 \%) \\
2(6.2 \%)\end{array}$ & $\begin{array}{c}15(31.3 \%) \\
32(66.7 \%) \\
1(2 \%)\end{array}$ & 0.486 \\
\hline Tiempo en HD (meses) & $21.75(8-42.4)$ & $18.5(7.5-39.3)$ & $25(8.5-45.5)$ & 0.207 \\
\hline Diabetes & $34(42.5 \%)$ & $15(46.9 \%)$ & $19(39.6 \%)$ & 0.518 \\
\hline Índice de Charlson & $6(4-8)$ & $6(4-7)$ & $7(4-8)$ & 0.426 \\
\hline IMC $\left(\mathrm{kg} / \mathrm{m}^{2}\right)$ & 25.9 (23.6-29.95) & $28(24.9-30.7)$ & 23.8 (22.3-29.2) & 0.067 \\
\hline $\mathrm{Kt} / \mathrm{V}$ & $1.43(1.2-1.61)$ & $1.37(1.13-1.56)$ & $1.49(1.27-1.65)$ & 0.16 \\
\hline Hemoglobina (g/dL) & $11.9(11-12.6)$ & $11.7(11-12.5)$ & $12(10.9-12.7)$ & 0.516 \\
\hline Leucocitos $\left(\times 10^{3} / \mathrm{mm}^{3}\right)$ & $6.5(5.2-7.6)$ & $6.7(5.2-7.6)$ & $6.3(5.2-7.5)$ & 0.848 \\
\hline Albúmina (g/dL) & $3.5(3.2-3.7)$ & $3.4(3.2-3.6)$ & $3.5(3.2-3.7)$ & 0.984 \\
\hline PCRus (mg/dL) & $0.75(0.3-1.2)$ & $0.9(0.4-1.3)$ & $0.6(0.3-1)$ & 0.047 \\
\hline INL & $2.7(1.7-3.6)$ & $3.3(2-4.4)$ & $2(1.3-3.1)$ & 0.039 \\
\hline IPL & $130.3(94.1-173.6)$ & $149.1(101.1-197.2)$ & $111.5(87.1-150)$ & 0.042 \\
\hline 25-OH-D (ng/mL) & $25.2(18.1-35.3)$ & $11.9(9.8-14.5)$ & $38.6(26.4-56.1)$ & $<0.01$ \\
\hline Ferritina (ng/mL) & $595.1(385.5-880.7)$ & 620.3 (401.9-932.1) & 569.9 (369.1-829.3) & 0.382 \\
\hline PTH-i (pg/mL) & $357.6(212.1-591.2)$ & 388.2 (208.8-668.9) & 326.9 (215.3-513.5) & 0.984 \\
\hline Calcio (mg/dL) & $8.4(8-8.9)$ & $8.3(8-8.8)$ & $8.5(8.1-9.1)$ & 0.13 \\
\hline Fósforo (mg/dL) & $4.7(4.2-5.9)$ & $4.6(4.1-5.7)$ & $4.8(4.3-6.2)$ & 0.669 \\
\hline c-LDL (mg/dL) & $78.3(54.2-100.2)$ & $83.5(60.8-102.5)$ & $73(47.5-98)$ & 0.252 \\
\hline c-HDL (mg/dL) & 37 (32.7-43.9) & 38 (32.3-45.3) & $36(33-42.5)$ & 0.552 \\
\hline Triglicéridos (mg/dL) & $116.5(84.2-158.8)$ & $115(83.5-147)$ & $118(85-170.5)$ & 0.869 \\
\hline $\begin{array}{l}\text { Tratamientos } \\
\text { Eritropoyetina } \\
\text { Antihipertensivos } \\
\text { Análogos de la vitamina D } \\
\text { Dosis EPO (U/semana) } \\
\text { Dosis de hierro (mg/semana) } \\
\text { IRE }\end{array}$ & $\begin{array}{c}79(98.8 \%) \\
57(71.2 \%) \\
59(73.8 \%) \\
8250(4000-15000) \\
33.3(25-81.25) \\
9.6(5-19.25)\end{array}$ & $\begin{array}{c}31(96.9 \%) \\
20(62.5 \%) \\
28(87.5 \%) \\
7500(4500-15000) \\
33.3(25-87.5) \\
10.5(4.7-18.8)\end{array}$ & $\begin{array}{c}48(100 \%) \\
37(77.1 \%) \\
31(64.6 \%) \\
9000(3500-15000) \\
33.3(12.5-75) \\
8.7(5.3-19.7)\end{array}$ & $\begin{array}{l}0.218 \\
0.158 \\
0.022 \\
0.722 \\
0.501 \\
0.798\end{array}$ \\
\hline
\end{tabular}

Test $\mathrm{U}$ de Mann-Whitney. $\mathrm{RI}$ = rango intercuartílico, 25-OH-D = 25-OH-vitamina $\mathrm{D}, \mathrm{IMC}=$ índice de masa corporal, $\mathrm{PCRus}=$ proteína $\mathrm{C}$ reactiva ultrasensible, INL = índice neutrófilo/ linfocito, IPL = índice plaqueta/linfocito, PTH-i = hormona paratiroidea intacta, c-LDL = colesterol de la lipoproteína de baja densidad, c-HDL = colesterol de la lipoproteína de alta densidad, EPO = eritropoyetina, IRE = índice de resistencia a la eritropoyetina.

una relación significativa entre los niveles de vitamina D y PCR. ${ }^{21}$ Adicionalmente, Yildirim et al. estudiaron la asociación de 25-OH-D con PCR, velocidad de sedimentación globular y leucocitos en población con y sin ERC y no identificaron asociación entre marcadores inflamatorios y $25-\mathrm{OH}-\mathrm{D}{ }^{22}$ 
Tabla 2. Estatus de vitamina D en pacientes en hemodiálisis $(N=80)$

\begin{tabular}{|l|c|c|}
\hline Deficiencia de vitamina D $(25-\mathrm{OH}-\mathrm{D}<20 \mathrm{ng} / \mathrm{mL})$ & 32 & 40 \\
\hline Deficiencia severa de vitamina D $(25-\mathrm{OH}-\mathrm{D}<10 \mathrm{ng} / \mathrm{mL})$ & 8 & 10 \\
\hline Insuficiencia de vitamina D $(25-\mathrm{OH}-\mathrm{D} 21-30 \mathrm{ng} / \mathrm{mL})$ & 16 & 20 \\
\hline $\begin{array}{l}\text { Suficiencia de vitamina D }(25-\mathrm{OH}-\mathrm{D}>30 \mathrm{ng} / \mathrm{mL}) \\
\text { 25-OH-D }=25-\mathrm{OH} \text {-vitamina D }\end{array}$ & 32 & 40 \\
\hline
\end{tabular}

Tabla 3. Correlación bivariada entre niveles de 25-OH-vitamina D y otros parámetros asociados $(\mathrm{N}=80)$

\begin{tabular}{|c|c|c|}
\hline Parámetro & $\begin{array}{l}\text { Coeficiente de correlación } \\
\text { de Spearman (ro) }\end{array}$ & p \\
\hline Edad (años) & 0.067 & 0.552 \\
\hline Tiempo en HD (meses) & 0.177 & 0.117 \\
\hline Índice de Charlson & 0.059 & 0.6 \\
\hline $\mathrm{IMC}\left(\mathrm{kg} / \mathrm{m}^{2}\right)$ & -0.153 & 0.175 \\
\hline $\mathrm{Kt} / \mathrm{v}$ & 0.245 & 0.034 \\
\hline Hemoglobina (g/dL) & 0.071 & 0.529 \\
\hline Leucocitos $\left(\times 10^{3} / \mathrm{mm}^{3}\right)$ & 0.08 & 0.481 \\
\hline Albúmina (g/dL) & 0.003 & 0.98 \\
\hline PCRus (mg/dL) & -0.334 & 0.026 \\
\hline INL & -0.523 & 0.013 \\
\hline IPL & -0.451 & 0.022 \\
\hline Ferritina (ng/mL) & 0.052 & 0.644 \\
\hline PTH-i (pg/mL) & -0.093 & 0.411 \\
\hline Calcio (mg/dL) & 0.197 & 0.08 \\
\hline Fósforo (mg/dL) & -0.063 & 0.579 \\
\hline $\mathrm{c}-\mathrm{LDL}(\mathrm{mg} / \mathrm{dL})$ & -0.052 & 0.662 \\
\hline $\mathrm{c}-\mathrm{HDL}(\mathrm{mg} / \mathrm{dL})$ & -0.073 & 0.525 \\
\hline Triglicéridos (mg/dL) & 0.097 & 0.395 \\
\hline Dosis EPO (U/semana) & -0.045 & 0.694 \\
\hline Dosis de hierro (mg/semana) & -0.072 & 0.526 \\
\hline IRE & -0.005 & 0.966 \\
\hline
\end{tabular}

25-OH-D = 25-OH-vitamina D, IMC = índice de masa corporal, PCRus = proteína C reactiva ultrasensible, INL = índice neutrófilo linfocito, IPL = índice plaqueta linfocito, PTH $-\mathrm{i}=$ hormona paratiroidea intacta, $\mathrm{c}-\mathrm{LDL}=$ colesterol de la lipoproteína de baja densidad, c-HDL = colesterol de la lipoproteína de alta densidad, EPO = eritropoyetina, $\mathrm{IRE}=$ índice de resistencia a la eritropoyetina.

En la población en HD son limitados los datos sobre la relación entre la vitamina $\mathrm{D}$ y los marcadores inflamatorios. Mirchi et al. analizaron la relación entre los niveles de 25-OH-D, INL y PCR, los cuales fueron significativamente más altos en el grupo con $25-\mathrm{OH}-\mathrm{D}<10 \mathrm{ng} / \mathrm{mL}$; encontraron una correlación inversa débil entre $25-\mathrm{OH}-\mathrm{D}$ y PCR. ${ }^{23} \mathrm{Kara}$ et al. estudiaron la asociación entre los niveles de vitamina $D$ y los marcadores inflamatorios e informaron una relación inversa estadísticamente significativa entre la vitamina D y los niveles de PCR e INL. ${ }^{24}$

Nuestra investigación respalda los hallazgos de los estudios mencionados y reveló una asociación inversa entre los niveles de 25-OH-D y los nuevos marcadores inflamatorios INL e IPL. Además, también se encontró una correlación positiva con el Kt/v, donde $K$ es el aclaramiento de urea en el dializador, $t$ es el tiempo de diálisis y v, el volumen de distribución de urea. Nessim et al. también demostraron una correlación entre los niveles de vitamina $D$ y Kt/v. ${ }^{25}$ Estos resultados sugieren un vínculo entre el aumento del aclaramiento urémico en HD y sus efectos metabólicos en la síntesis de vitamina D. La evidencia experimental indica que las toxinas urémicas pueden interferir en la síntesis cutánea de vitamina $D$, en forma análoga a la melanina acumulada en el estrato de Malpighi, donde ocurre la síntesis de vitamina D. ${ }^{26}$

Un $\mathrm{Kt} / \mathrm{v}<1.2$ se asocia con estado inflamatorio crónico que resulta en altos niveles de PCR. ${ }^{27}$ Borazán et al. reportaron una correlación negativa entre los niveles séricos de $\mathrm{PCR}$ y Kt/v en pacientes en $\mathrm{HD}$ $(r=-0.436, p<0.05) .{ }^{28}$ Por otro lado, Herzig et al. describieron esta correlación en pacientes en diálisis peritoneal $(r=-0.30, p<0.05){ }^{29}$

La deficiencia de vitamina $D$ puede derivar en hiperparatiroidismo secundario en individuos con función renal normal. Sin embargo, la relación entre la hormona paratiroidea y la $25-\mathrm{OH}-\mathrm{D}$ ha sido menos clara en los ensayos clínicos que evalúan a pacientes en diálisis. En nuestro estudio, no se encontró asociación significativa entre los niveles de 25-OH-D, fósforo, calcio y hormona paratiroidea. Adicionalmente, en otras investigaciones tampoco se encontró correlación entre los niveles de vitamina $D$ y los parámetros del metabolismo óseo. ${ }^{19,30}$ Por el contrario, el estudio observacional de Ojeda et al. realizado en pacientes en HD con déficit de 25-OH-D evidenció descenso en los niveles de PCR y hormona paratiroidea después de tres meses de tratamiento con calcifediol. ${ }^{31}$

En el estudio de Ahmadi et al., ${ }^{5}$ la deficiencia de vitamina $\mathrm{D}$ se asoció significativamente con obesidad central; en nuestro análisis, los pacientes con deficiencia de vitamina $D$ tuvieron un índice de masa corporal 
más alto, pero las diferencias no fueron significativas. Encontramos que los pacientes con $25-\mathrm{OH}-\mathrm{D}<20 \mathrm{ng} / \mathrm{mL}$ tenían un nivel de albúmina más bajo en comparación con los pacientes con niveles $\geq 20 \mathrm{ng} / \mathrm{mL}$, pero la diferencia no fue significativa. En el estudio de Bansal et al. ${ }^{32}$ se encontró una correlación negativa débil entre los niveles de 25-OH-D y el índice de masa corporal, sexo y albúmina en los pacientes en HD.

Nuestros pacientes con deficiencia de vitamina $D$ presentaron niveles de hemoglobina más bajo y de índice de resistencia a la eritropoyetina más altos, sin embargo, las diferencias no fueron estadísticamente significativas. Una razón podría ser que los objetivos terapéuticos respecto a la anemia en los pacientes en HD han sido cumplidos en nuestra unidad. A su vez, se ha descrito que uno de los factores que más influye en la resistencia a la eritropoyetina es el estado inflamatorio, ${ }^{33}$ el cual en nuestro estudio fue mayor en el grupo con deficiencia de vitamina $D$.

Nuestro trabajo presenta limitaciones. El estudio fue de corte transversal, por lo tanto, no podemos establecer una relación causal entre los marcadores inflamatorios y los niveles séricos de vitamina $\mathrm{D}$. Los datos obtenidos presentan limitaciones por el hecho de que las mediciones bioquímicas se realizaron solo una vez, lo que no tiene en cuenta los cambios dependientes del tiempo. El tamaño de la muestra fue relativamente pequeño, aunque el número de pacientes fue adecuado para mostrar la significación estadística de las correlaciones. Otra limitación fue el número reducido de marcadores inflamatorios estudiados, si bien los seleccionados son ampliamente utilizados, económicos y probados.

\section{Conclusiones}

Encontramos que $40 \%$ de los pacientes presentó deficiencia de vitamina $\mathrm{D}$. Adicionalmente, el estudio mostró que los valores de PCRus, INL e IPL fueron significativamente mayores en el grupo con deficiencia de vitamina $D$, mientras que $\mathrm{Kt} / \mathrm{v}$ fue significativamente menor en este grupo. Mostramos una correlación inversa significativa entre los niveles séricos de vitamina $D$ y los valores de PCRus, INL e IPL, todos marcadores de inflamación económicos y universalmente disponibles. Los resultados pueden sugerir que existe un mecanismo subyacente desconocido responsable de la relación inversa observada en los pacientes en HD. Nuestro estudio proporciona una contribución significativa a la literatura, en la cual en general se han informado resultados controvertidos sobre el tema; creemos que puede conducir a otras investigaciones más completas que investigarán mejor la relación entre los niveles de vitamina $\mathrm{D}$ y los marcadores inflamatorios en la población en HD.

\section{Conflicto de intereses}

Ninguno.

\section{Fuentes de financiamiento}

La presente investigación no ha recibido ninguna beca específica de agencias de los sectores público, comercial, o sin ánimo de lucro.

\section{Responsabilidades éticas}

Protección de personas y animales. Los autores declaran que para esta investigación no se realizaron experimentos en seres humanos ni en animales.

Confidencialidad de los datos. Los autores declaran que siguieron los protocolos de su centro de trabajo sobre la publicación de datos de pacientes.

Derecho a la privacidad y consentimiento informado. Los autores declaran que en este artículo no aparecen datos de pacientes.

\section{Bibliografía}

1. Blair D, Byham-Gray L, Lewis E, McCaffrey S. Prevalence of vitamin D [25(OH)D] deficiency and effects of supplementation with ergocalciferol (vitamin D2) in stage 5 chronic kidney disease patients. J Ren Nutr. 2008;18(4):375-382.

2. Bataille S, Landrier JF, Astier J, Giaime P, Sampol J, Sichez H, et al. The "dose-effect" relationship between 25 -hydroxyvitamin D and muscle strength in hemodialysis patients favors a normal threshold of $30 \mathrm{ng} / \mathrm{mL}$ for plasma 25-hydroxyvitamin D. J Ren Nutr. 2016;26:45-52.

3. Lee YH, Kim JE, Roh YH, Choi HR, Rhee Y, Kang DR, et al. The combination of vitamin $D$ deficiency and mild to moderate chronic kidney disease is associated with low bone mineral density and deteriorated femoral microarchitecture: results from the KNHANES 2008-2011. J Clin Endocrinol Metab. 2014:99:3879-388.

4. Boudville N, Inderjeeth C, Elder G, Glendenning P. Association between 25 -hydroxyvitamin $\mathrm{D}$, somatic muscle weakness and falls risk in end-stage renal failure. Clin Endocrinol (Oxf). 2010;73:299-304.

5. Ahmadi F, Damghani S, Lessan-Pezeshki M, Razeghi E, Maziar S, Mahdavi-Mazdeh M. Association of low vitamin D levels with metabolic syndrome in hemodialysis patients. Hemodial Int. 2016;20:261-269.

6. Shaffi K, Tighiouart H, Scott T, Lou K, Drew D, Weiner D, et al. Low 25-hydroxyvitamin $D$ levels and cognitive impairment in hemodialysis patients. Clin J Am Soc Nephrol. 2013;8:979-986.

7. Drechsler C, Pilz S, Obermayer-Pietsch B, Verduijn M, Tomaschitz A, Krane $\mathrm{V}$, et al. Vitamin $\mathrm{D}$ deficiency is associated with sudden cardiac death, combined cardiovascular events, and mortality in haemodialysis patients. Eur Heart J. 2010;31:2253-2261.

8. Lai S, Coppola B, Dimko M, Galani A, Innico G, Frassetti N, et al. Vitamin $D$ deficiency, insulin resistance, and ventricular hypertrophy in the early stages of chronic kidney disease. Ren Fail. 2014;36:58-64.

9. Mukai H, Villafuerte $H$, Qureshi AR, Lindholm B, Stenvinkel P. Serum albumin, inflammation, and nutrition in end-stage renal disease: C-reactive protein is needed for optimal assessment. Semin Dial. 2018;31: 435-439.

10. Heidari B. C-reactive protein and other markers of inflammation in hemodialysis patients. Casp J Intern Med. 2012;4:611-616. 
11. Valga F, Monzón T, Henríquez F, Antón-Pérez G. Índices neutrófilo-linfocito y plaqueta-linfocito como marcadores biológicos de interés en la enfermedad renal. Nefrologia. 2019;39:243-249.

12. Shaarawy A, Baki AH, Teama NM, Abdel Halim RM, Eldin Fahim NA Sultan RA. Neutrophils to lymphocytes ratio is an easy non expensive marker of inflammation in hemodialysis patients. J Clin Exp Nephrol. 2018;3:0-6

13. Mangin M, Sinha R, Fincher K. Inflammation and vitamin D: the infection connection. Inflamm Res. 2014;63:803-819.

14. Mohiuddin SA, Marie M, Ashraf M, Hussein M, Almalki N. Is there an association between Vitamin D level and inflammatory markers in hemodialysis patients? A cross-sectional study. Saudi J Kidney Dis Transpl. 2016;27:460-466.

15. Daugirdas JT. Second generation logarithmic estimates of single-pool variable volume $\mathrm{Kt} / \mathrm{V}$ : an analysis of error. J Am Soc Nephrol. 1993:4:1205-1213.

16. Holick MF, Binkley NC, Bischoff-Ferrari HA, Gordon CM, Hanley DA, Heaney RP, et al. Evaluation, treatment, and prevention of vitamin D deficiency: an endocrine society clinical practice guideline. J Clin Endocrinol Metab. 2011;96:1911-1930.

17. Eknoyan G, Levin A, Levin NWB. Clinical practice guidelines for bone metabolism and disease in chronic kidney disease: an overview. Am J Kidney Dis. 2003;42:S1-S201.

18. Ahbap E, Sakaci T, Kara E, Sahutoglu T, Koc Y, Basturk T, et al. Neutrophil-to-lymphocyte ratio and platelet-to-lymphocyte ratio in evaluation of inflammation in end-stage renal disease. Clin Nephrol. 2016;85:199-208.

19. Marinelli A, Pistolesi V, Rossi V, Battista M, Buono A, Della-Grotta F, et al. Severe $25-\mathrm{OH}$ vitamin D deficiency in patients on chronic hemodialysis. G Ital Nefrol. 2014;31:8-15.

20. Akbas EM, Gungor A, Ozcicek A, Akbas N, Askin S, Polat M. Vitamin D and inflammation: evaluation with neutrophilto-lymphocyte ratio and platelet-to-lymphocyte ratio. Arch Med Sci. 2016;12:721-727.

21. Grzanka A, Machura E, Mazur B, Misiolek M, Jochem J, Kasperski J, et al. Relationship between vitamin D status and the inflammatory state in patients with chronic spontaneous urticaria. Eur $J$ Dermatol. 2015;25:26-28.
22. Yildirim I, Hur E, Kokturk F. Erythrocyte sedimentation rate, and leukocyte count in vitamin D deficient patients with and without chronic kidney disease. Int J Endocrinol. 2013;2013:8021.

23. Mirchi E, Saghafi H, Gharehbeglou M, Aghaali M, Rezaian Z, Ghaviahd M. Association between 25-hydroxyvitamin D level and inflammatory and nutritional factors in hemodialysis and peritoneal dialysis patients in Qom, Iran. Iran J Kidney Dis. 2016;10:205-212.

24. Kara AV, Soylu YE. The relationship between vitamin $D$ and inflammatory markers in maintenance hemodialysis patients. Int Urol Nephrol. 2019;51:1659-1665.

25. Nessim SJ, Jassal SV, Fung SV, Chan CT. Conversion from conventional to nocturnal hemodialysis improves vitamin D levels. Kidney Int. 2007;71:1172-1176.

26. Holick MF. Vitamin D and the kidney. Kidney Int. 1987;32:912-929.

27. Rashid H, Rizwan-ul-Haq, Abad-ur-Rehman. Comparison of C-reactive protein levels with delivered dose of $\mathrm{Kt} / \mathrm{V}$ in patients with end-stage renal disease on maintenance hemodialysis. Saudi J Kidney Dis Transpl. 2015;26:692.

28. Borazan A, Aydemir S, Sert M, Yilmaz A. The effects of hemodialysis and peritoneal dialysis on serum homocysteine and $\mathrm{C}$-reactive protein levels. Mediators Inflamm. 2004;13:361-364.

29. Herzig KA, Purdie DM, Chang W, Brown AM, Hawley CM, Campbell SB, et al. Is C-reactive protein a useful predictor of outcome in peritoneal dialysis patients? J Am Soc Nephrol. 2001;12:814-821.

30. Coen G, Mantella D, Manni M, Balducci A, Nofroni I, Sardella D, et al. 25-hydroxyvitamin $\mathrm{D}$ levels and bone histomorphometry in hemodialysis renal osteodystrophy. Kidney Int. 2005;68:1840-1848.

31. Ojeda-López R, Esquivias-de Motta E, Carmona A, García-Montemayor V, Berdud I, Martín-Malo A, et al. La corrección del déficit de 25-OH-vitamina D mejora el control del hiperparatiroidismo secundario y el estado inflamatorio de pacientes estables en hemodiálisis. Nefrologia. 2018;38:41-47.

32. Mithal A, Kher V, Marwaha R, Bansal B, Bansal S. Vitamin D deficiency in hemodialysis patients. Indian J Endocrinol Metab. 2012;16:270-273.

33. Icardi A, Paoletti E, de Nicola L, Mazzaferro S, Russo R, Cozzolino M. Renal anaemia and EPO hyporesponsiveness associated with vitamin D deficiency: the potential role of inflammation. Nephrol Dial Transplant. 2013;28(7):1672-1679 